\title{
Efeito dos métodos de fotoativação e de inserção sobre a dureza de resinas compostas
}

\section{Effect of the methods of photoactivation and insertion on the hardness of composite resins}

\author{
Simonides Consani* \\ Samira Buselli Pereira** \\ Mario Alexandre Coelho Sinhoreti* \\ Lourenço Correr Sobrinho*
}

\begin{abstract}
RESUMO: Este trabalho verificou o efeito do tipo de inserção (duas camadas ou bloco único) e dos métodos de fotoativação (luz contínua, duplo pulso ou intermitente), sobre a dureza Knoop dos compósitos Z100 e Alert. Os corpos-de-prova foram confeccionados em cavidades de $4 \times 4 \mathrm{~mm}$, com o material inserido de duas maneiras diferentes: porção única ou em duas camadas de $2 \mathrm{~mm}$ de espessura cada uma. O material inserido numa única porção foi comprimido com carga estática de $1 \mathrm{kgf}$ exercido sobre uma lâmina de vidro, interposta por uma tira de poliéster, para remoção do excesso. Depois de retirar a lâmina de vidro e a tira de poliéster, a fotoativação foi por luz contínua emitida pelo aparelho XL 3000, com intensidade de $520 \mathrm{~mW} / \mathrm{cm}^{2}$, por 40 segundos; duplo pulso, 10 segundos com emissão de $150 \mathrm{~mW} / \mathrm{cm}^{2}$ mais 30 segundos com $520 \mathrm{~mW} / \mathrm{cm}^{2}$ com o aparelho XL 3000; e luz pulsátil de $520 \mathrm{~mW} / \mathrm{cm}^{2} \mathrm{emitida}$ pelo aparelho Optilux 400, sendo 2 segundos ligado e 1 segundo desligado, por 60 segundos. As duas camadas da inserção dupla foram fotoativadas nas mesmas condições da inserção única, assim como a remoção do excesso de material da última camada. Após armazenagem em estufa a $37^{\circ} \mathrm{C}$ e umidade relativa de $100 \%$ por 24 horas, os corpos-de-prova foram embutidos em resina de poliéster, desgastados e submetidos ao polimento com lixas de granulação decrescente e pasta de diamante. A dureza Knoop foi avaliada em diferentes profundidades com penetrômetro HMV Shimadzu, sob carga de $50 \mathrm{~g}$ durante 30 segundos. Os resultados submetidos à análise de variância e ao teste de Tukey (5\%) mostraram que o Z100 apresentou maior valor de dureza; a inserção dupla foi melhor que a inserção única e a dureza de superfície foi maior que nas demais profundidades, independente dos demais fatores. No Z100 todos os métodos de fotoativação promoveram maior dureza na inserção dupla, enquanto no Alert a ativação por luz contínua promoveu similaridade estatística entre os dois tipos de inserção. O Z100 mostrou similaridade estatística entre os métodos de fotoativação somente na inserção dupla, enquanto no Alert a similaridade estatística ocorreu na inserção única entre as ativações por duplo pulso e luz pulsátil.
\end{abstract}

DESCRITORES: Resinas compostas; Restauração dentária permanente; Dureza.

\begin{abstract}
The purpose of this study was to assess the effect of methods of photoactivation and insertion on the Knoop hardness of the $\mathrm{Z} 100$ and Alert composite resins. The specimens were confected in cavities measuring $4 \times 4 \mathrm{~mm}$. The insertion of material was carried out by means of two methods: single-portion technique and insertion of two 2-mm-thick layers. When inserted in a single portion, the resin was compressed with a static load of $1 \mathrm{kgf}$ on a glass slab recovered with a polyester strip, in order to remove the excess of material. After the removal of the glass slab and polyester strip, the materials were photoactivated by means of continuous light emitted by a XL 3000 unit with a light intensity of $520 \mathrm{~mW} / \mathrm{cm}^{2}$ for 40 seconds; double pulse, with light emission of $150 \mathrm{~mW} / \mathrm{cm}^{2}$ for 10 seconds, plus 30 seconds with light intensity of $520 \mathrm{~mW} / \mathrm{cm}^{2}$ emitted by a XL 3000 unit; and pulsatile light of $520 \mathrm{~mW} / \mathrm{cm}^{2} \mathrm{emitted}$ by the Optilux 400 unit, turned on for 2 seconds and off for 2 seconds, during 60 seconds. The two layers of the material submitted to double insertion were photoactivated in the same conditions as the bulk-inserted material, and the excess of material was also removed from the second layer. After storage in a stove at $37^{\circ} \mathrm{C}$ and $100 \%$ relative humidity for 24 hours, the specimens were embedded in polyester resin, trimmed and polished with sandpaper and diamond slurry. Knoop hardness was assessed in 4 depths with a HMV Shimadzu penetrometer under the load of $50 \mathrm{~g}$ during 30 seconds. The data submitted to ANOVA and Tukey's test revealed that Z100 presented greater hardness values; double insertion was better than single insertion; the hardness at the surface was smaller than that at the bottom of the specimen, regardless of other factors. For Z100, all activation methods produced smaller hardness values when double insertion was carried out. Meanwhile, for Alert, continuous light promoted statistical similarity between the insertion techniques. For Z100, there was statistical similarity between the activation methods only when the double-insertion technique was employed. There was statistical similarity between the double-pulse and pulsatile-light techniques for Alert, when the single-portion insertion technique was carried out.
\end{abstract}

DESCRIPTORS: Composite resins; Dental restoration, permanent; Hardness.

*Professores da Área Materiais Dentários; **Aluna de Graduação - Faculdade de Odontologia de Piracicaba da Universidade Estadual de Campinas. 
Consani S, Pereira SB, Sinhoreti MAC, Correr Sobrinho L. Efeito dos métodos de fotoativação e de inserção sobre a dureza de resinas compostas. Pesqui Odontol Bras 2002;16(4):355-360.

\section{INTRODUÇÃO}

Os compósitos são usados na Odontologia há mais de 30 anos e têm sofrido constantes modificações por parte dos fabricantes, proporcionando melhores propriedades mecânicas e resultados estéticos mais satisfatórios aos pacientes ${ }^{6,7,11,16}$.

Os materiais atuais são complexos e tecnicamente sofisticados. Assim, para evitar que a seleção seja efetuada somente de acordo com a preferência do dentista, torna-se importante compreender as propriedades físicas, mecânicas e biológicas, assim como o comportamento clínico desses restauradores estéticos ${ }^{12}$. A morfologia e o tamanho das partículas têm grande influência nas diversas propriedades do material, como lisura de superfície, viscosidade, resistência à fratura, desgaste, dureza, contração e profundidade de polimerização ${ }^{1,4,5,10}$.

No início, os compósitos eram ativados por reação química, posteriormente por ativação física através da luz ultravioleta e atualmente pela luz visivel, emitida por aparelhos fotopolimerizadores, responsáveis pela excitação das substâncias fotossensiveis, que reagem com o agente iniciador da reação ${ }^{13,14,19,20,27}$.

O sistema de luz visível apresenta inúmeras vantagens sobre os sistemas anteriormente utilizados $^{23}$; entretanto, embora a taxa de polimerização tenha sido melhorada ainda continua insuficiente, sendo considerada uma das principais causas do insucesso clínico. Por outro lado, o grau da polimerização também pode ser influenciado pelo direcionamento da luz, condições técnicas do aparelho, tamanho das partículas e cor do material $^{8,9}$.

A intensidade de luz emitida pelos fotopolimerizadores tem sido considerada um dos fatores mais importantes no desempenho dessas unidades, uma vez que a variação dos valores da intensidade de luz poderia promover alterações significantes na taxa de polimerização dos compósitos, causando deficiência de adaptação marginal, na resistência da união do compósito ao dente e na dureza de superfície ${ }^{3,18}$.

Atualmente, a maioria dos aparelhos fotopolimerizadores encontrados no mercado exibem intensidade de luz em torno de $500 \mathrm{~mW} / \mathrm{cm}^{2}$. Isto leva a crer que a maioria dos problemas relacionados com a deficiência de polimerização devido à baixa intensidade de luz estaria solucionado. No entanto, uma das características inerente dos compósitos odontológicos impondo restrições às técnicas restauradoras é a contração de polimerização. A taxa de contração de polimerização é alta e promove tensão na região da união dente-restauração, criando espaço que certamente será propício à invasão de fluidos bucais e bactérias ${ }^{15}$.

A técnica de inserção do compósito na cavidade é muito importante e procura-se por meio dela minimizar o efeito da contração de polimerização. A inserção do compósito em camadas horizontais ou inclinadas é o artifício de técnica mais utilizado para diminuir os efeitos da contração de polimerização ${ }^{17}$. Rueggeberg et al. ${ }^{24}$ (1994) verificaram que em profundidades maiores que $2 \mathrm{~mm}$ a polimerização era incompleta e causada pelas mudanças na intensidade luminosa e no tempo de exposição. Assim, a espessura das camadas incrementais não devia ultrapassar $2 \mathrm{~mm}$ para que a polimerização fosse completa, não afetando a dureza e outras propriedades do material.

De acordo com Koran, Kürschner ${ }^{18}$ (1998), o método de polimerização pode também ser um artifício no controle dessa contração, por meio da ativação inicial em baixa intensidade de luz, seguida por posterior exposição com alta intensidade. Segundo o autor, essa técnica promoverá melhor adaptação marginal da restauração, devido ao relaxamento das tensões ocorrido no compósito durante a fase da polimerização inicial. Porém, nesse caso, a taxa de exposição à luz fica diminuída, podendo interferir no grau de polimerização do compósito e, conseqüentemente, afetar a dureza do material.

Este estudo propõe verificar o efeito da inserção do compósito em camadas ou bloco único e dos métodos de ativação por luz contínua, duplo pulso e por luz intermitente, sobre os valores de dureza Knoop dos compósitos odontológicos Z100 e Alert, com diferentes conteúdos de carga.

\section{MATERIAL E MÉTODOS}

Neste estudo foram utilizados os compósitos restauradores $Z 100(3 \mathrm{M})$, com carga de zircônia-sílica e matriz orgânica de Bis-GMA e TEGMA e Alert (Jeneric/Pentron), com alto conteúdo de carga contendo vidro de borossilicato de bário-sílica, dióxido de sílicio, óxidos de magnésio, alumínio e ferro, e matriz orgânica composta de policarbonato DMA, DAEM e DMA difenol-A etoxilato.

Os corpos-de-prova foram confeccionados numa matriz acrílica contendo uma cavidade com $4 \mathrm{~mm}$ de diâmetro por $4 \mathrm{~mm}$ de espessura, secionada longitu- 
Consani S, Pereira SB, Sinhoreti MAC, Correr Sobrinho L. Efeito dos métodos de fotoativação e de inserção sobre a dureza de resinas compostas. Pesqui Odontol Bras 2002;16(4):355-360.

dinalmente na parte central, a fim de facilitar a remoção do corpo-de-prova após a polimerização.

Com auxílio de uma espátula plástica, o compósito foi inserido na cavidade de duas maneiras diferentes, ou seja, numa única porção ou em duas camadas, com $2 \mathrm{~mm}$ de espessura cada uma. Na inserção única, o material foi comprimido com carga estática de $1 \mathrm{kgf}$ exercido sobre uma lâmina de vidro interposta por uma tira de poliéster, com a intenção de remover o excesso de material e deixar a superfície do compósito nivelado à borda da parede lateral da matriz. Depois de retirar o peso, a lâmina de vidro e a tira de poliéster, os corpos-de-prova foram fotoativados de acordo com um dos métodos propostos: luz contínua emitida pelo aparelho XL 3000, com intensidade de $520 \mathrm{~mW} / \mathrm{cm}^{2}$, por 40 segundos; duplo pulso, com espaçador de $12 \mathrm{~mm}$ de comprimento adaptado na ponteira do aparelho XL 3000, para que a emissão de luz fosse de $150 \mathrm{~mW} / \mathrm{cm}^{2}$ por $10 \mathrm{se}$ gundos, seguido de 30 segundos a $520 \mathrm{~mW} / \mathrm{cm}^{2}$, sem espaçador; e luz pulsátil de $520 \mathrm{~mW} / \mathrm{cm}^{2}$ emitida pelo aparelho Optilux 400 especialmente adaptado para esse propósito, sendo 2 segundos ligado e 1 segundo desligado, até completar 60 segundos. As duas camadas de material da técnica de inserção dupla foram fotoativadas nas mesmas condições da inserção única, assim como a remoção do excesso de material da última camada.

Após confecção, os corpos-de-prova foram identificados, demarcados com caneta hidrográfica na superfície de incidência da luz fotoativadora e armazenados em estufa a $37^{\circ} \mathrm{C}$ e $100 \%$ de umidade relativa, por 24 horas. Cinco corpos-de-prova foram confeccionados para cada grupo, de acordo com o método de fotoativação e tipo de inserção, totalizando 30 repetições para cada material.

Decorridas 24 horas, os corpos-de-prova foram fixados verticalmente em posição lateral numa placa de vidro com adesivo à base de cianoacrilato (Super Bonder - Loctite) e embutidos em resina (Poliéster 5060 MP, Ramires \& Cia. Ltda., Taboão da Serra - SP), previamente preparada na relação resina/ativador de $10 \mathrm{~g} / 1,5 \mathrm{ml}$. Após polimerização, a resina foi desgastada numa politriz (APC-2, Arotec), sob constante irrigação com água, inicialmente com lixas d'água de granulações decrescentes de 220, 320, 400, 500, 600 e 1000, finalizando com feltro e pasta de diamante $(0,5 \mu \mathrm{m})$.

A superficie polida dos corpos-de-prova foi submetida ao teste de dureza Knoop nas regiões: $30 \mu \mathrm{m}$ abaixo da superfície de incidência direta da luz, nas regiões intermediárias de $1,5 \mathrm{~mm}$ e $2,5 \mathrm{~mm}$ de pro- fundidade e na região de fundo. As medidas de dureza foram efetuadas com penetrômetro HMV 2000 Shimadzu (Japão), sob carga de 50 gramas, por 30 segundos. Quatro penetrações foram efetuadas em cada região de análise, totalizando 16 penetrações para cada corpo-de-prova. Oitenta penetrações foram efetuadas em cada um dos seis grupos envolvendo os dois compósitos, totalizando 960 penetrações no ensaio.

Os valores obtidos em micrômetros foram convertidas em número de dureza Knoop por meio de programa de software e submetidos à análise de variância com esquema fatorial, cujos fatores foram: material, que analisou o compósito restaurador; método de fotoativação, que analisou o método de ativação; método de inserção, que analisou o tipo de inserção; e região, que analisou a profundidade de polimerização. Os valores médios foram submetidos ao teste de Tukey em nível de 5\% de probabilidade.

\section{RESULTADOS}

A análise de variância mostrou que independente dos fatores inserção, fotoativação e profundidade, o compósito $Z 100$ obteve valores médios de dureza Knoop $(96,2 \pm 1,4)$ com diferença estatística significativa $(\mathrm{p}<0,05)$ quando comparado com o Alert $(84,8 \pm 1,0)$. Os valores médios de dureza Knoop para tipos de inserção, independente do material, fotoativação e profundidade foram maiores e estatisticamente significantes para a inserção dupla $(95,8 \pm 1,0)$ quando comparado à porção única $(85,8 \pm 1,4)$.

Independente dos demais fatores, os métodos de fotoativação diferiram estatisticamente entre si e a maior média foi obtida pela luz contínua $(94,4 \pm 1,3)$, seguida pelo duplo pulso $(91,1 \pm 1,4)$ e luz pulsátil $(85,9 \pm 1,8)$. A dureza na região da incidência de luz $(99,0 \pm 1,0)$ foi superior e diferiu estatisticamente das regiões de $1,5 \mathrm{~mm}$ $(96,2 \pm 1,1), 2,5 \mathrm{~mm}(92,1 \pm 1,6)$ e fundo $(74,6 \pm 1,7)$, as quais também diferiram estatisticamente entre si.

O Gráfico 1 mostra a comparação entre os valores médios de dureza Knoop do compósito Z100 em função do tipo de inserção e método de fotoativação. Houve diferença estatística significativa entre os três métodos de ativação somente na inserção única. Houve diferença estatística significativa entre os tipos de inserção em todos métodos de fotoativação. 
Consani S, Pereira SB, Sinhoreti MAC, Correr Sobrinho L. Efeito dos métodos de fotoativação e de inserção sobre a dureza de resinas compostas. Pesqui Odontol Bras 2002;16(4):355-360.

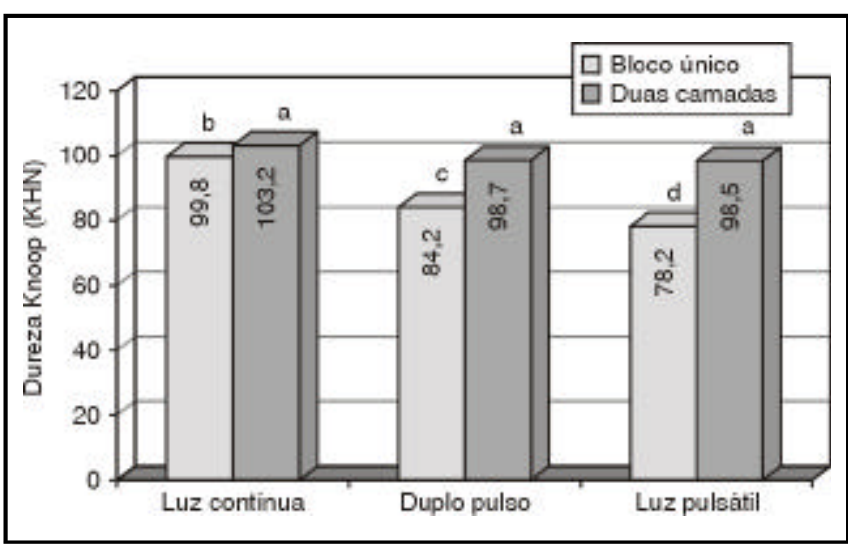

GRÁFICO 1 - Valores médios de dureza Knoop do compósito Z100, em função do tipo de inserção e método de fotoativação. Barras agrupadas ou da mesma cor identificadas por letras distintas diferem entre si $(5 \%)$.

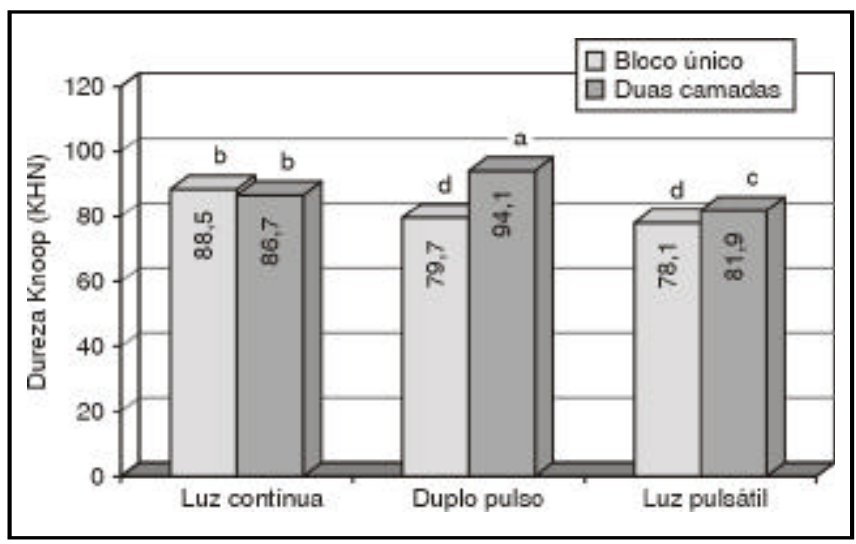

GRÁFICO 2 - Valores médios de dureza Knoop do compósito Alert, em função do tipo de inserção e método de fotoativação. Barras agrupadas ou da mesma cor identificadas por letras distintas diferem entre si (5\%).

Os valores médios de dureza Knoop do compósito Alert em função do tipo de inserção e método de fotoativação são mostrados no Gráfico 2. Não houve diferença estatística significativa entre os tipos de inserção somente no método de fotoativação por luz contínua. Dentro do fator método de fotoativação, não houve diferença estatística significativa somente entre o duplo pulso e a luz pulsátil na inserção única.

\section{DISCUSSÃO}

Independente dos fatores inserção, fotoativação e profundidade de polimerização, o compósito $Z 100$ apresentou valor médio de dureza Knoop maior e com diferença estatística significativa quando comparado com o obtido pelo Alert. Isso poderia ter ocorrido em função da composição dos materiais. O Z100 é um compósito formulado com carga híbrida, constituída de partículas de zircônio-sílica, com maior dureza devido aos vidros cerâmicos, de formato semi-esférico e tamanho médio de $0,6 \mu \mathrm{m}$, conferindo-lhe baixa resistência ao cisalhamento. Assim, o fabricante consegue inserir maior quantidade de carga sem afetar a aplicabilidade clínica devido ao escoamento. Já, o Alert apresenta maior resistência ao cisalhamento em função da predominância da carga de vidro de borossilicato de alumínio e bário, acrescido de cargas filamentares de carbono, o que dificulta o escoamento.

A técnica da porção dupla apresentou resultados médios de dureza superiores e com diferença estatística significativa quando comparado ao da técnica de porção única, independente dos materiais, fotoativação e profundidade de polimerização. Como mostrado na literatura, quanto mais espessa for a camada, maior será a dificuldade para converter monômero em polímero, o que diminui a dureza ${ }^{9,11}$. Segundo Rueggeberg et al. ${ }^{24}$ (1994), os incrementos devem ter no máximo $2 \mathrm{~mm}$ de espessura para que a polimerização seja completa e uniforme, sendo que o ideal seria uma espessura de $1 \mathrm{~mm}$. Inadequada polimerização modifica as propriedades do compósito, como dureza, taxa de absorção de água, solubilidade, estabilidade de cor, comprometendo a resistência e a estética da restauração. Por outro lado, polimerização incompleta do material na base da restauração pode também promover falha da retenção e causar danos à polpa dental ${ }^{22}$.

A dureza Knoop do Z100 (Gráfico 1) mostrou resultados com diferença estatística significativa entre as técnicas de fotoativação somente na inserção única, sempre inferior ao da porção dupla em cada método de fotoativação. A dureza do Alert (Gráfico 2) mostrou diferença estatística significativa entre todos os métodos de fotoativação apenas na inserção dupla, com maior valor para o duplo pulso. Na inserção única as fotoativações por duplo pulso e luz pulsátil mostraram similaridade estatística entre si e ambas diferiram estatisticamente da fotoativação por luz contínua, com maior valor de dureza. Quando se considera que a inserção dupla foi duplamente ativada em todos os métodos de fotoativação, a similaridade estatística dos valores de dureza ocorrida somente na luz contínua parece indicar ser este o único método fotoativador capaz de concordar com a informação do fabricante de que o Alert pode ser usado em cama- 
Consani S, Pereira SB, Sinhoreti MAC, Correr Sobrinho L. Efeito dos métodos de fotoativação e de inserção sobre a dureza de resinas compostas. Pesqui Odontol Bras 2002;16(4):355-360.

das de até $5 \mathrm{~mm}$, sem comprometer as propriedades mecânicas.

O método da luz contínua apresentou o maior valor médio de dureza Knoop, com diferença estatística significativa quando comparado com os demais, independente do material, fotoativação e profundidade de polimerização. O método da luz pulsátil apresentou o menor valor médio de dureza Knoop e uma possivel explicação seria que o aparelho realizou ciclos de 3 segundos, sendo 2 segundos com luz acesa e 1 segundo com luz apagada. Conseqüentemente, quando a luz era ligada não atingia imediatamente a intensidade máxima do aparelho $\left(520 \mathrm{~mW} / \mathrm{cm}^{2}\right)$, permanecendo com baixa intensidade no primeiro instante, somente atingindo a intensidade máxima pouco antes de completar o ciclo. Como o tempo em que o aparelho permaneceu nessa intensidade foi curto, a eficácia ativadora da luz seria na repetição contínua do ciclo, embora, o somatório da intensidade fosse considerado insuficiente para uma adequada polimerização. Entretanto, os corpos-de-prova polimerizados por este método provavelmente ficaram sujeitos a menores taxas de energia de luz, causando menor dureza ao material.

A profundidade de polimerização pode ser afetada por vários fatores, incluindo a intensidade de luz, tempo de exposição, distância do fotoativador e quantidade de absorção de luz pelos compósitos ${ }^{21,26,28}$. De acordo com Pires et al. ${ }^{23}$ (1993), a distância do fotoativador ao compósito deve ser de $1 \mathrm{~mm}$, embora a superfície seja menos dependente da distância do que o fundo da amostra.

Houve um decréscimo estatisticamente significativo em profundidade dos valores das médias de dureza Knoop nas regiões superfície, 1,5 mm, $2,5 \mathrm{~mm}$ e fundo, independente da inserção, material e fotoativação. A luz ativa o fotoiniciador na superfície, começando a reação para conversão do monômero em polímero. Continuando a exposição, ocorre a ativação das moléculas do fotoiniciador próximo à superfície. Entretanto, a absorção e a reflexão da luz pelo compósito diminuem a sua intensidade abaixo da superfície, tornando mais críticas a velocidade e a taxa de conversão, conseqüentemente, reduzindo a dureza ${ }^{2}$. Confirmando esse fato, autores como De Lange et al. ${ }^{11}$ (1980); Rueggeberg et al. ${ }^{24}$ (1994); Correr Sobrinho ${ }^{9}$ (1995) verificaram que quanto mais espessa for a camada, menor será a dureza do compósito nas regiões mais profundas.

O maior valor de dureza Knoop foi obtido próximo à superfície ( $30 \mu \mathrm{m}$ de profundidade), com diferença estatística significativa quando comparado com as demais regiões. Segundo Unterbrink, Muessner ${ }^{25}$ (1995), os valores máximos de dureza são verificados a $0,5 \mathrm{~mm}$ abaixo da camada superficial do compósito, região livre de material com polimerização incompleta devido ao oxigênio que reage com a cadeia de radicais livres ${ }^{15}$.

O método de fotoativação por duplo pulso apresentou menor valor médio de dureza Knoop, com diferença estatística significativa quando comparado com a maior média promovida pela luz contínua. Este resultado pode ser devido à igualdade do tempo empregado para fotoativação nos dois métodos (40 segundos). No método por duplo pulso, a intensidade da luz foi de $150 \mathrm{~mW} / \mathrm{cm}^{2}$ nos primeiros 10 segundos, seguida por 30 segundos a $520 \mathrm{~mW} / \mathrm{cm}^{2}$, foi insuficiente para compensar a baixa taxa de polimerização inicial imposta ao material. O recurso técnico compensatório para melhorar a dureza seria aumentar o tempo de fotoativação com intensidade alta, pois muitos estudos $^{19,20,23,27}$ mostraram que a fotoativação por duplo pulso promoveria adaptação marginal mais satisfatória, sem comprometer a dureza.

O material Alert com porção dupla (Gráfico 2) determinou maior valor de dureza Knoop para o método de duplo pulso com inserção dupla, com diferença estatística significativa quando comparado com os da luz contínua e luz pulsátil, confirmando que a quantidade de intensidade luminosa que atinge o material interfere na taxa de polimerização. Entretanto, no material Z100 (Gráfico 1) não houve diferença estatística significativa entre os três métodos de fotoativação na inserção dupla, onde o aumento da intensidade luminosa para polimerizar as duas camadas foi suficiente para promover similar dureza no material polimerizado em todos os métodos de ativação.

\section{CONCLUSÃO}

O compósito $Z 100$ apresentou maior valor médio de dureza, a dupla inserção promoveu melhores resultados que a inserção única e a dureza na região de superfície foi maior que nas demais profundidades, independente dos demais fatores. No Z100 todos os métodos de fotoativação promoveram maior dureza na inserção dupla, enquanto no Alert a ativação por luz contínua promoveu similaridade estatística entre os dois tipos de inserção. $\mathrm{O}$ Z100 mostrou similaridade estatística entre os métodos de fotoativação somente na inserção dupla, enquanto no Alert a similaridade estatística ocorreu na inserção única entre as ativações por duplo pulso e luz pulsátil. 


\section{REFERÊNCIAS}

1. Anusavice KJ. Phillips' science of dental materials. $10^{\text {th }}$ ed. Philadelphia: W.B. Saunders; 1996.

2. Asmussen E. Restorative resins: hardness and strength versus quantity of remaining double bonds. Scand J Dent Res 1982;90(6):484-9.

3. Atmadja G, Bryant RW. Some factors influencing the depth of cure of visible-light-activated composite resins. Austr Dent J 1990;35(3):213-8.

4. Bassiouny MA, Grant AA. A visible light-cured composite restorative: clinical open assessment. Br Dent $\mathrm{J}$ 1978;145(11):327-30.

5. Beatty MW, Swartz ML, Moore BK, Phillips RW, Roberts TA. Effect of microfiller fraction and silane treatment on resin composite properties. J Biomed Mater Res 1998;40(1):12-23.

6. Blankenau RJ, Kelsey WP, Cavel WT, Blankenau P. Wavelength and intensity of seven systems for visible-light-curing composite resins: a comparison study. J Am Dent Assoc 1983;106(4):471-4.

7. Cook WD, Standish PM. Cure of resin-based restorative materials. II - white light photopolymerized resins. Austr Dent J 1983;28(5):307-311.

8. Coradazzi JL, Francichone CE, Franco EB. Avaliação de um aparelho com lâmpada halógena para polimerização de resinas compostas. Rev Paul Odontol 1986;8(4):50-4.

9. Correr Sobrinho L. Correlação intensidade de luz-tempo de exposição sobre a polimerização de compósitos odontológicos. [Tese de Doutorado] Ribeirão Preto: Faculdade de Odontologia de Ribeirão Preto da USP; 1995.

10. De Backer J, Dermaut L. Visible light sources and posterior light cures resins: a practical mixture. Quintessence Int 1986;17(10):635-41.

11. De Lange C, Bausch JR, Davidson CL. The curing pattern of photo-initiated dental composites. J Oral Rehabil 1980;7(5):369-77.

12. Denyer R, Shaw DJ. Cure evaluation of visible light composites by Knoop hardness measurement [abstract n.833]. J Dent Res 1982;61(1):271.

13. Dune SM, Davies BR, Millar BJ. A survey of the effectiveness of dental light-curing units and a comparison of light testing devices. Br Dent J 1996;180(11):411-6.

14. Eliades GC, Vougioulklankis GJ, Caputo AA et al. Degree of double bond conversion in light-cured composites. Dent Mater 1987;3:19-25.
15. Fraunhofer JA. The surface hardness of polymeric restorative materials. Br Dent J 1971;130(16):243-5.

16. Galan Júnior J, Langhi M, Castellanos V. Profundidade de polimerização de resinas compostas ativadas por luz. Rev Paul Odontol 1984;6(6):27-9.

17. Hirabayashi S, Hood JÁ, Hirasawa T. The extent of polymerization of class II light-cured composite resin restorations: effects of incremental placement technique, exposure time and heating for resin inlays. Dent Mater J 1993;12(2):159-70.

18. Koran P, Kürschner R. Effect of sequential versus continuous irradiation of a light-cure resin composite on shrinkage, viscosity, adhesion and degree of polymerization. Am J Dent 1998;11(1):17-22.

19. Mehl A, Manhart J, Hickel R et al. The influence of pre-curing on the material properties of composite resins [abstract n.496]. J Dent Res 1995;74(3):462.

20. Mehl A, Hickel R, Kunzelmann RH et al. Physical properties and gap formation of light-cured composites with and without "softstart-polymerization". J Dent 1997;25(3-4):321-30.

21. Pilo R, Oelgiesser D, Cardash HS. A survey of output intensity and potential for depth of cure among light-curing units in clinical use. J Dent 1999;27:235-41.

22. Pires JAF, Cvitko E, Denehy GE et al. Effects of curing tip distance on light intensity and composite resin microhardness. Quintessence Int 1993;24(7):517-21.

23. Pollack BF, Blitzer MH. The advantages of visible light-curing resins. N Y State Dent J 1982;48:228-30.

24. Rueggeberg FA, Caughman WF, Curtis Júnior JW et al. Effect of light intensity and exposure duration on cure of composite resin. Oper Dent 1994;19(1):26-32.

25. Unterbrink GL, Muessner R. Influence of light intensity on two restorative systems. J Dent 1995:23(3):183-9.

26. Vicentini A, Correr Sobrinho L, Consani S et al. Fotopolimerização das resinas compostas. Influência da intensidade de luz e do tempo de exposição no grau de dureza Knoop. RGO. 1997;44(3):146-8.

27. Watts DC, Amer OM, Combe EC. Characteristics of visible light-activated composite systems. Br Dent J 1984;156(6):209-15.

28. Yearn JA. Factors affecting cure of visible-light-activated composites. Int Dent J 1985;35(3):218-25. 